\title{
Dynamics in Supercooled Secondary Amide Mixtures: Dielectric and Hydrogen Bond Specific Spectroscopies - Supporting Information
}

\author{
C. Gainaru, ${ }^{1, \#}$ S. Bauer, ${ }^{1}$ E. Vynokur, ${ }^{1}$ H. Wittkamp, ${ }^{1}$ W. Hiller, ${ }^{2}$ R. Richert, ${ }^{3}$ R. Böhmer ${ }^{1}$ \\ ${ }^{1}$ Fakultät Physik, Technische Universität Dortmund, 44221 Dortmund, Germany \\ ${ }^{2}$ Fakultät Chemie, Technische Universität Dortmund, 44221 Dortmund, Germany \\ ${ }^{3}$ School of Molecular Sciences, Arizona State University, Tempe, Arizona 85287-1604, USA
}

\section{Dielectric experiments}

To provide a direct comparison of the dielectric relaxation times we measured and analyzed spectra for $(\mathrm{NMA})_{0.4}(\mathrm{NMF})_{0.6},(\mathrm{NMA})_{0.2}(\mathrm{NEA})_{0.2}(\mathrm{NMF})_{0.6}$, and $(\mathrm{NEA})_{0.4}(\mathrm{NMF})_{0.6}$. The results for the time constants characterizing the Debye peak are displayed in Fig. S1, see also Fig. 8 of ref. 1. This figure demonstrates that addition of NMA slows down the Debye dynamics in these mixtures, although its molecular size is smaller than the one of NEA.

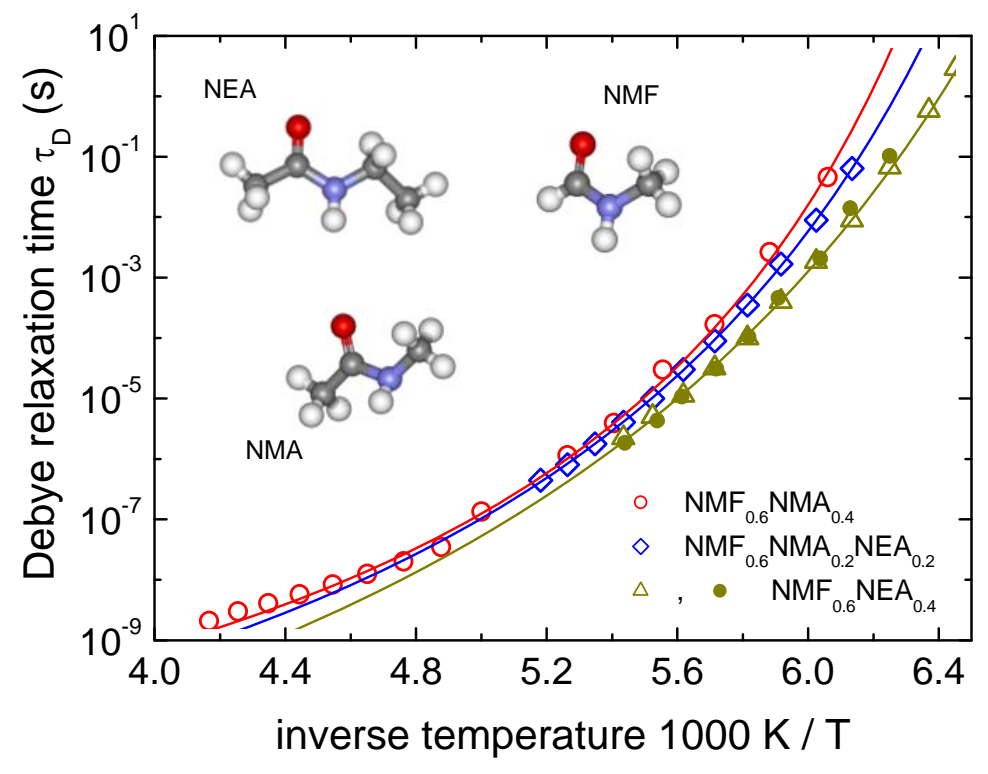

Fig. S1 Dielectric time scales of the Debye process in mixtures of NMF with NEA or NMA as specified. All data are from the current work except those for (NMA) ${ }_{0.4}(\mathrm{NMF})_{0.6}$ plotted as dots which are taken from ref. 2. Solid lines are calculated using eq. (4) of ref. 1. The inset depicts the molecular structures of the investigated liquids. The red spheres correspond to oxygen atoms and the blue ones to nitrogen atoms.

\# Current address: Department of Chemistry, Tennessee University, Knoxville, TN 37996-1600, USA 


\section{Field gradient diffusometry}

Employing a home-built spectrometer and using standard Hahn-echo as well as stimulated-echo pulse sequences ${ }^{3}$ we measured the translational diffusion coefficient $D_{t}$ of fully protonated (NMA) $)_{0.4}(\mathrm{NMF})_{0.6}$. The proton NMR measurements were carried out $\omega_{\mathrm{L}}=2 \pi$ $\times 53.4 \mathrm{MHz}$ in a static field gradient $g$ of $\sim 19.9 \mathrm{~T} / \mathrm{m}$. The results from both data sets could be well described using the Vogel-Fulcher law $D_{t}(T)=9.3 \times 10^{-8} \mathrm{~m} / \mathrm{s}^{2} \cdot \exp [-1063 \mathrm{~K} /(T-91 \mathrm{~K})]$. From the diffusion coefficients presented in Fig. S2 we calculated the time constants $\tau_{\text {Diff }}$ that are presented in Fig. 8 of ref. 1. For comparison diffusion coefficients for NMF and $N, N$ dimethylformamide (DMF) are included in Fig. S2. ${ }^{4}$ Expectedly, due to a lack of hydrogen bonding, the latter compounds exhibits the fastest diffusion.

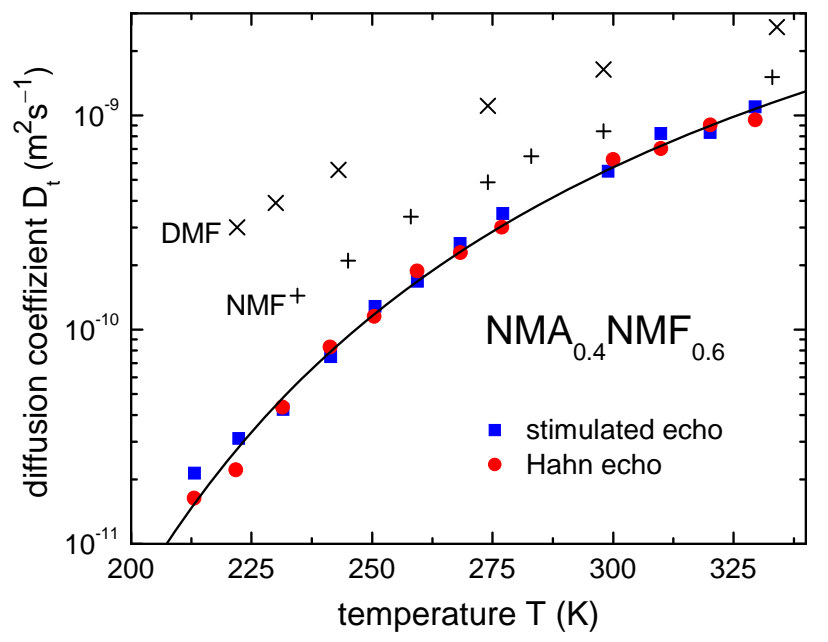

Fig. S2 Temperature dependent translational diffusion coefficient of $(\mathrm{NEA})_{0.4}(\mathrm{NMF})_{0.6}$ as determined using various echo methods applied in the present work. The line reflects the fit described in the text. The data for NMF and DMF are from ref. 4.

\section{Proton and nitrogen chemical-shift experiments}

Using a DPX-300 NMR spectrometer from Bruker BioSpin, chemical-shift experiments were carried out at magnetic field of $7 \mathrm{~T}$ corresponding to ${ }^{1} \mathrm{H}$ and ${ }^{15} \mathrm{~N}$ Larmor frequencies of 300 and $30.4 \mathrm{MHz}$, respectively. As seen from Fig. S3 a large temperature range was covered. In the $\mathrm{NH}$ region the proton resonances are split due to spin-spin coupling with the $\mathrm{CH}_{3}$ and the $\mathrm{CH}_{2}$ groups which is most pronounced near temperatures of about 210 to $220 \mathrm{~K}$. As a consequence a quartet appears for the NH signal of NMF and a triplet for the 
N-H signal of NEA (not shown). To check this assignment we carried out a homonuclear twodimensional correlation spectroscopy experiment at $215 \mathrm{~K}$ and show the result as Fig. S4.

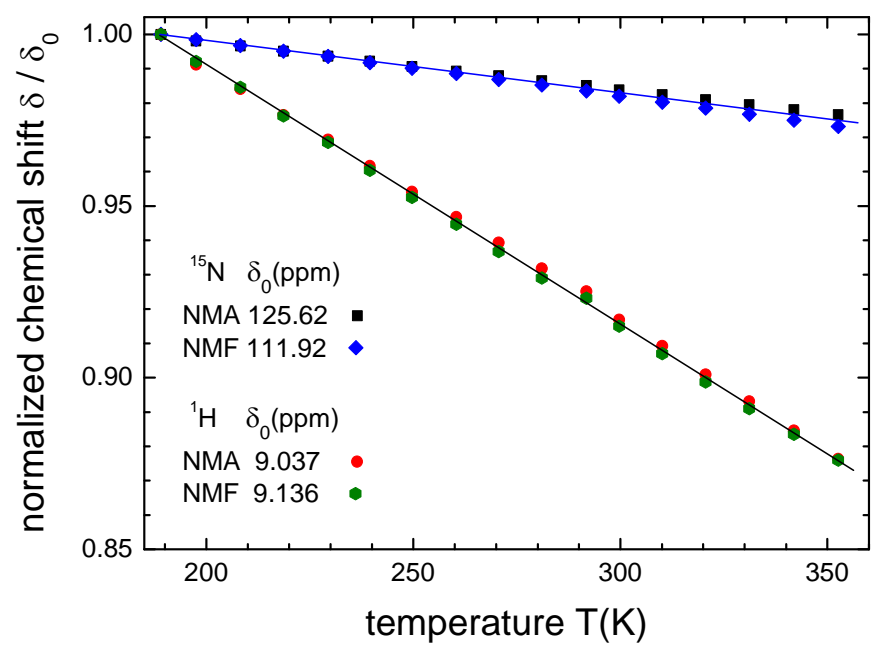

Fig. S3 Temperature dependent chemical shifts of $\mathrm{NEA}_{0.5} \mathrm{NMF}_{0.5}$ measured for the $\mathrm{NH}$ groups. The data - scaled to their values $\delta_{0}$ at $189 \mathrm{~K}$ - are compatible with a linear behavior over the entire temperature range. The reference values $\delta_{0}$ are measured with respect to the high-field triplet of the methyl groups of NEA (which in turn appears near $1.3 \mathrm{ppm}$ ).

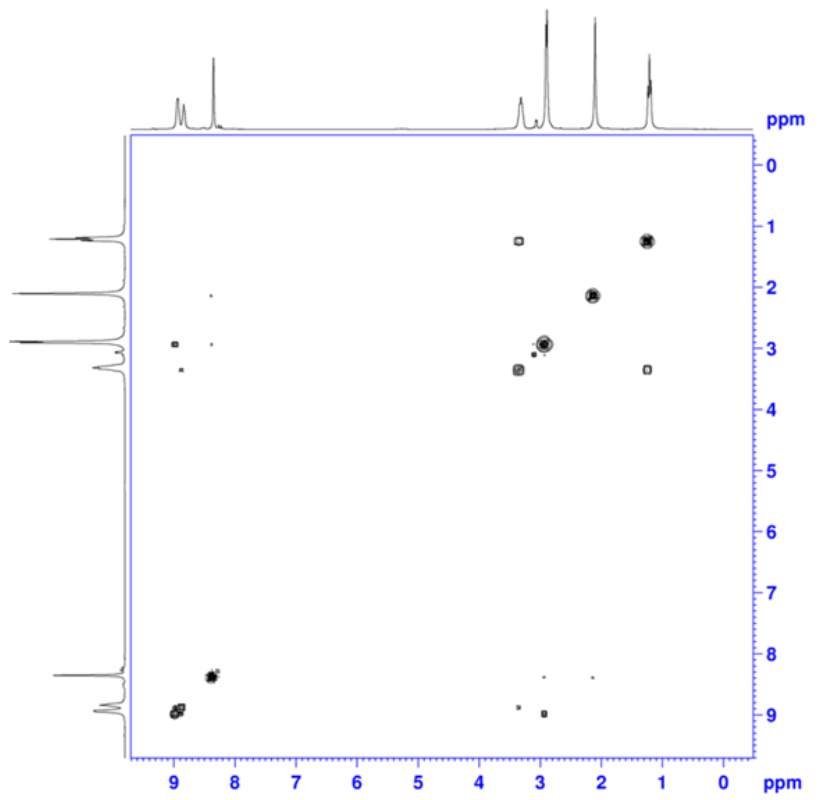

Fig. S4 Two-dimensional ${ }^{1} \mathrm{H}^{-1} \mathrm{H}$ chemical shift correlation spectrum (COSY) of $\mathrm{NEA}_{0.5} \mathrm{NMF}_{0.5}$ recorded at $215 \mathrm{~K}$. Three correlations are indicated: Apart from the coupling of the $\mathrm{CH}_{2}-\mathrm{CH}_{3}$ groups of NEA, the coupling of the $\mathrm{NH}$ to the $\mathrm{CH}_{2}$ group of NEA is also seen as well as the coupling of the NMF's NH group to its $\mathrm{CH}_{3}$ group. The cross peaks thus allows for an unambiguous distinction of the two different $\mathrm{NH}$ groups. The two cross peaks belonging to the signal at $8.41 \mathrm{ppm}$ are artifacts arising from the symmetrization of the spectra. 
Near $T \approx 270 \mathrm{~K}$ the strongly temperature dependent proton resonances of the $\mathrm{NH}$ groups overlap with the essentially temperature independent proton resonance of NMF's aldehyde group. This circumstance then precludes a reliable determination of chemical shift positions. Therefore, to be selectively sensitive to the $\mathrm{NH}$ protons, for $T \geq 270 \mathrm{~K}$ we carried out heteronuclear ${ }^{15} \mathrm{~N}-{ }^{1} \mathrm{H}$ two-dimensional correlation measurements. As seen in Fig. S5 this allowed us to determine the chemical shifts precisely, also at higher temperatures.

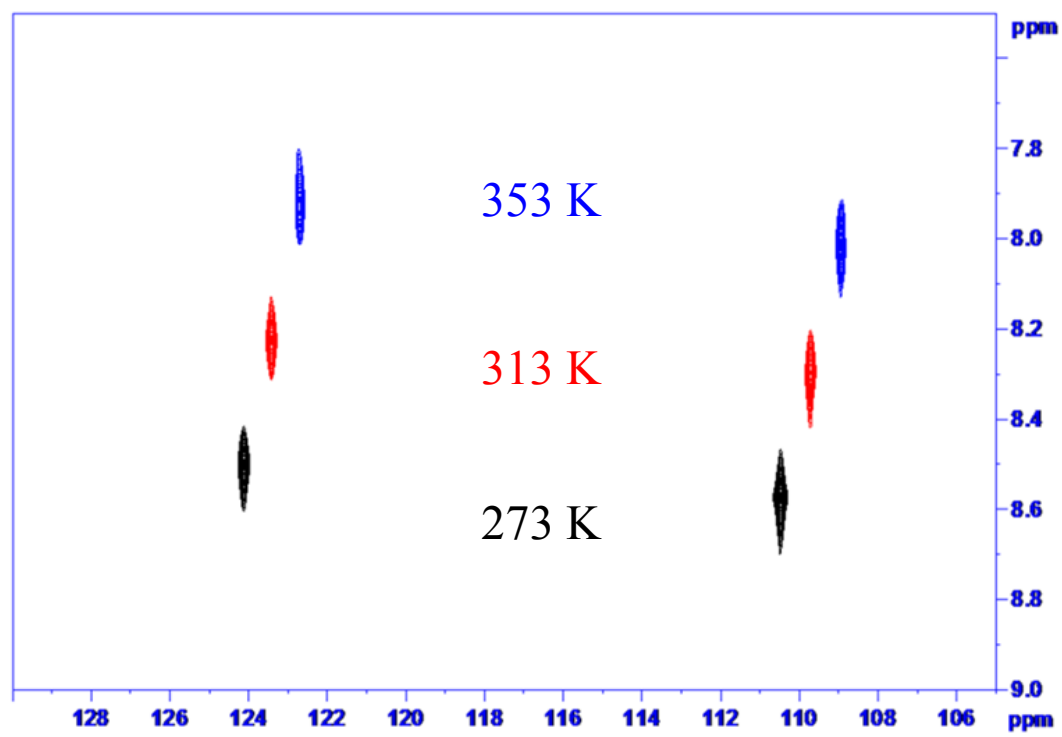

Fig. S5 Two-dimensional correlation (HETCOR) of ${ }^{15} \mathrm{~N}$ chemical shifts (on the $\mathrm{x}$-axis) versus ${ }^{1} \mathrm{H}$ chemical shifts (on the $\mathrm{y}$-axis) which allows for an unambiguous assignment of the proton resonances of the $\mathrm{NH}$ groups as well as the ${ }^{15} \mathrm{~N}$ resonances in $\mathrm{NEA}_{0.5} \mathrm{NMF}_{0.5}$. The shifts of the ${ }^{15} \mathrm{~N}$ resonance that are larger than $120 \mathrm{ppm}$ refer to NEA, those at smaller $\delta$ to NMF. Data are shown for temperatures of 273,313 , and $353 \mathrm{~K}$ as indicated.

\section{References}

1 Gainaru, C.; Bauer, S.; Vynokur, E.; Wittkamp, H.; Hiller, W.; Richert, R.; Böhmer, R. Dynamics in Supercooled Secondary Amide Mixtures: Dielectric and Hydrogen Bond Specific Spectroscopies. J. Phys. Chem. B (http://dx.doi.org/10.1021/acs.jpcb.5b10034).

2 Wang, L. M.; Richert, R. Identification of Dielectric and Structural Relaxations in Glass Forming Secondary Amides. J. Chem. Phys. 2005, 123, 054516.

3 Lederle, C.; Hiller, W.; Gainaru, C.; Böhmer, R. Diluting the Hydrogen Bonds in Viscous Solutions of n-Butanol with n-Bromobutane: II. A Comparison of Rotational and Translational Motions. J. Chem. Phys. 2011, 134, 064512.

4 Easteal, A. J.; Wolf, L. A. Self-Diffusion and Volumetric Measurements for $\mathrm{N}$ Methylformamide and $N, N$-Dimethylformamide at Temperatures from 240 to $313 \mathrm{~K}$ and Pressures up to $300 \mathrm{MPa}$. J. Chem. Soc., Faraday Trans. 1 1985, 81, 2821-2833. 